\title{
Production of anti-Ro/SS-A and anti-La/SS-B Autoantibodies is Closely Coordinated in Systemic Lupus Erythematosus and Independent of anti-dsDNA Production
}

\author{
Jan F. Meilof ${ }^{1}$, Charles H. A. Veldhoven ${ }^{1}$, Anton J. G. Swaak ${ }^{2}$ and Ruud J. T. Smeenk ${ }^{1}$
}

${ }^{1}$ Dept. of Autoimmune Diseases, Central Laboratory of the Netherlands Red Cross Blood Transfusion Service, Amsterdam, NL-1066 CX. The Netherlands

${ }^{2}$ Dept. of Rheumatology, Daniel Den

Hoed Clinic, Rotterdam, NL-3075 EA,

The Netherlands

Received 5 February 1996

Accepted 18 October 1996

Key words: SLE, anti-Ro/SS-A, anti-La/SS-B, anti-DNA, autoimmunity, scRNP tolerance
Autoimmune diseases such as Sjögren's Syndrome and systemic lupus erythematosus (SLE) are characterized by serum autoantibodies against protein components of small cytoplasmic ribonucleoproteins (scRNPs). The origin and regulation of these anti-Ro/SS-A and anti-La/SS-B antibodies is not well understood. Regular co-occurrence of these two autoantibodies in humans together with murine studies on antibody responses against scRNPs after immunization suggest a role for scRNPs as common antigen. We sought additional evidence for this hypothesis in a longitudinal serological study of patients with SLE. Quantitative measurement of the antibody responses against Ro/SS-A and La/SS-B proteins and for comparison to dsDNA in 852 serum samples was performed. These samples were collected from nine patients during an average observation period of more than 10 years. A significant and strong correlation between the two anti-scRNP responses emerged during $90 \%$ of follow-up. In contrast, correlation of anti-scRNP with anti-dsDNA responses was remarkably absent in the same patients. Our results confirm the unique relationship between anti-Ro/SS-A and anti-La/ SS-B responses and could thus be interpreted as support for a model wherein induction and perpetuation of autoantibody production is dependent on scRNPs containing both proteins as antigen.

\section{Introduction}

Autoantibody formation against self antigens is one of the core features of autoimmune diseases such as Systemic Lupus Erythematosus (SLE) [1, 2]. Breakdown of tolerance results in autoantibodies reacting with dsDNA and protein antigens such as small nuclear ribonucleoproteins (snRNPs, e.g. anti-Sm, anti-nRNP antibodies) or small cytoplasmic ribonucleoproteins (scRNPs, e.g. anti-Ro/SS-A and anti-La/SS-B antibodies) [3]. The former are well known to be correlated with active nephritis in patients with SLE $[4,5]$, the latter are not restricted to patients with SLE but are also found in sera of patients with Sjögren's syndrome [6, 7].

Anti-Ro/SS-A and anti-La/SS-B autoantibodies are amongst the few of their kind thought to be directly pathogenic: transplacental transfer of these antibodies from mother to unborn child damages the fetal cardiac conduction system resulting in congenital heart block $[8,9]$.

Correspondence to: Dr R. J. T. Smeenk.

Present address J. F. Meilof: Dept. of Neurology, Academic Medical Centre, Amsterdam, NL-1105 AZ, The Netherlands.
Different mechanisms have been proposed to explain the development of non-organ specific autoantibodies. These include polyclonal B cell activation and (auto)antigen driven responses [10] Several lines of evidence suggest that the latter mechanism is implicated in eliciting anti-Ro/SS-A and anti-La/SS-B antibodies. Firstly, anti-scRNP antibodies are predominantly of the IgG1 subclass, suggesting a T cell dependent (antigen driven) mechanism [11-13]. Secondly, epitope-mapping studies of Ro/SS-A and La/SS-B autoantigens have yielded results compatible with a sequence of events whereby the autoantigens themselves serve as driving force behind the initiation of the autoimmune response [14-16]. Thirdly, human specific epitopes have been unequivocally demonstrated on the La/SS-B protein [17].

Recent studies have proposed a new model for the development of autoantibodies especially those against small nuclear ribonucleoproteins (snRNPs) $[18,19]$. In these experiments only mice primed with human snRNPs developed a true B and T cell autoimmune response when immunized with murine snRNPs. The abrogation of $\mathrm{B}$ cell tolerance was 
necessary to allow the initiation of a $\mathrm{T}$ cell response against murine specific epitopes on snRNPs. These studies have now been extended, with similar results, to scRNP antigens [20].

Anti-Ro/SS-A antibodies in patients with SLE or Sjögren's Syndrome are very often accompanied by anti-La/SS-B antibodies and vice versa [21, 22]. This phenomenon could be explained by hypothesizing a common (auto)antigenic particle as driving force behind both autoantibody responses. Such a particle already exists in vivo: scRNPs consisting of Ro/SS-A and La/SS-B proteins coupled to so-called yRNAs (y1-y5 in humans) [23, 24]. If, however, scRNPs are the autoantigens responsible for eliciting anti-Ro/ SS-A and anti-La/SS-B autoantibodies, one would expect that, as most scRNP particles contain both proteins at the same time [25], the two antibody responses would parallel each other when followed in time. This hypothesis also explains the results of the immunization experiments resulting in both antibody specificities even when immunizing with only one recombinant antigen [20]. In search of further support for the validity of these murine studies, we decided to study the anti-Ro/SS-A and anti-La/SS-B antibody responses during longterm follow-up of patients with SLE. For this purpose we had at our disposal a unique collection of sera of SLE patients who were closely followed and very frequently sampled (approximately once per month) for up to 15 years [26]. Quantitative measurement of autoantibodies, using ELISAs based on purified recombinant proteins recently developed in our laboratory [27], indeed demonstrated a strong correlation between anti-Ro/SS-A and anti-La/SS-B responses. These responses were mostly dependent on the production of anti-dsDNA antibodies in the same patients.

\section{Methods}

\section{Human sera}

All patients selected fulfilled the revised ARA criteria for SLE [2]. Furthermore, for this study anti-Ro/SS-A and anti-La/SS-B autoantibodies had to be present in their circulation at some moment during follow-up and a large number of longitudinally collected serum samples had to be available. Using these criteria a panel of nine patients could be composed from a group of approximately 110 SLE patients visiting the rheumatology outpatient clinic of the Daniel Den Hoed Clinic in Rotterdam, The Netherlands.

\section{Recombinant Ro/SS-A and La/SS-B proteins}

Cloning and expression of human Ro/SS-A and La/SS-B proteins has been described elsewhere [27]. Briefly, the cDNAs encoding the $60 \mathrm{kDa}$ Ro/SS-A and $50 \mathrm{kDa}$ La/SS-B proteins were isolated using oligonucleotide primers based on the sequence previously published [28, 29]. Both cDNAs were cloned into the
pGEX7 expression system which resulted in a relatively high expression of the Ro/SS-A and La/SS-B proteins. Recombinant human Ro/SS-A and La/SS-B proteins were purified from bacterial pellets using sucrose-gradients, Phast-Q columns and HeparinSepharose columns. Purified protein preparations were frozen in liquid nitrogen and stored in small aliquots at $-70^{\circ} \mathrm{C}$ until use. Both preparations showed only a single band on Coomassie blue staining of gels.

\section{ELISAS}

ELISAs to measure antibody levels against recombinant $60 \mathrm{kDa}$ Ro/SS-A and $50 \mathrm{kDa} \mathrm{La} / \mathrm{SS}-\mathrm{B}$ proteins were performed as previously described [27]. Briefly, purified recombinant proteins were coated at $2 \mu \mathrm{g} / \mathrm{ml}$ onto microtitre plates (Nunc, Maxisorp) for 16 hours at $4^{\circ} \mathrm{C}$. Coated wells were incubated with sera serially diluted in PBS $(0.01 \mathrm{M}$ sodium phosphate, $\mathrm{pH} 7.4$, $0.14 \mathrm{M} \mathrm{NaCl}$ ) containing $0.02 \%$ Tween $20(\mathrm{v} / \mathrm{v}), 0.2 \%$ gelatin $(\mathrm{w} / \mathrm{v})$, and $3 \mathrm{M} \mathrm{NaCl}$ for $90 \mathrm{~min}$, after which the plates were washed 3 times. To detect bound human IgG, the wells were incubated with a mouse monoclonal anti-human IgG antibody (CLB-MH-16 E, binding to all four subclasses of IgG) coupled to horseradish peroxidase (HRP). The plates were then washed five times and developed with 3,5,3',5'tetramethylbenzidine (Merck). ELISA binding units were defined as the reciprocal serum dilution producing an OD value of 1.0 at $450 \mathrm{~nm}$. Cutoff values were determined by testing 100 healthy blood bank donors. This resulted in a cutoff value of 500 units for both ELISAs for human sera.

\section{Farr assay}

Antibodies to dsDNA were measured using the Farr assay as described previously [30]. Units were calculated upon comparison with the first international standard for antibodies to dsDNA: Wo80 [31]. Based on large series of controls, levels of more than $20 \mathrm{IU} /$ $\mathrm{ml}$ were considered positive.

\section{Total IgG1 determination}

Quantitation of $\operatorname{IgG}$ was performed as described previously using a sandwich ELISA protocol [32]. Briefly, plates were coated with a monoclonal antibody against IgG1 (MH161, prepared at the CLB) at $5 \mu \mathrm{g} /$ $\mathrm{ml}$. Bound immunoglobulins were detected using the same monoclonal antibody against IgG coupled to horseradish-peroxidase. Resulting titres were compared to well characterized reference sera to calculate the amount of IgG1 in the test sample.

\section{Statistics}

Data were analysed using non-parametric statistical methods: Spearman Rank Correlation test for paired observations. 

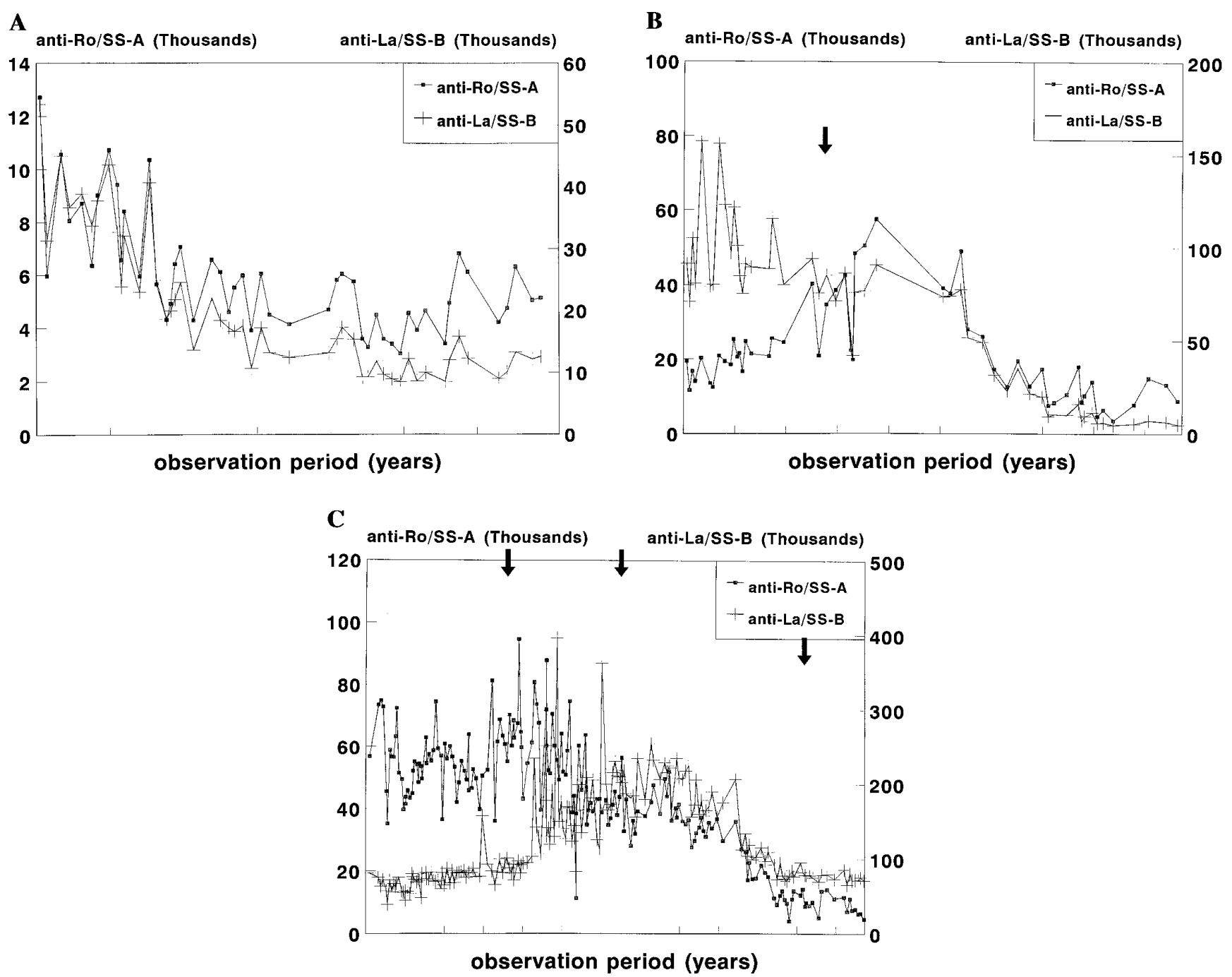

Figure 1. Development of anti-Ro/SS-A and anti-La/SS-B antibody levels in time. Antibodies against Ro/SS-A and La/SS-B were quantified using ELISAs. Reactivities, expressed in (thousands of) arbitrary units as described in the Methods section, are on the left (anti-Ro/SS-A) and right (anti-La/SS-B) y-axis. The observation period (in years) is on the $\mathrm{x}$-axis. (A) Patient $\mathrm{B}$ was followed for more than 7 years. Both anti-Ro/SS-A and anti-La/SS-B reactivities showed several fluctuations but followed each other closely. However, anti-La/SS-B antibodies displayed a 5-fold stronger reactivity. As in all other patients studied both antibody responses were already present at the start of the observation period, the moment the diagnosis SLE was established. (B) Patient G was followed for almost 10 years during which time anti-La/SS-B reactivities were twice as high as anti-Ro/SS-A reactivities most of the time. (C) Patient D was followed for almost 13 years during which period frequent serum samples were taken and stored. Although anti-Ro/SS-A and anti-La/SS-B reactivities sometimes fluctuated quite strongly, especially during the later years of observation, a more gradual pattern of decline to a base level occurs. Subdivision into periods for calculating correlation between the two responses (see legend to Figure 3) is marked by arrows.

\section{Results}

\section{Longitudinal development of anti-Ro/SS-A and anti-La/SS-B autoantibodies in sera of patients with SLE}

Anti-Ro/SS-A and anti-La/SS-B antibody levels fluctuated during the 91 patient years constituting the observation period. Examples of autoantibody profiles are depicted in Figure 1 (A, B and C). In patient B (Figure 1A) both antibody levels decreased during a number of years and then stabilized at a lower level.
In patient $\mathrm{G}$ (Figure 1B) anti-Ro/SS-A antibody levels first rose during a 3 year period before a relatively sharp drop to a much lower level. Anti-La/SS-B antibodies in this patient showed a different profile: a gradual decline to a low but still clearly positive base level. Finally in patient D (Figure 1C) the situation is almost reversed: anti-Ro/SS-A antibodies, after an initial small rise, gradually decreased in titre. AntiLa/SS-B antibodies in this patient in the end returned to the baseline level observed during the first years, after tripling to a broad peak reactivity spanning several years. In the other six patients studied, comparable profiles of autoantibody reactivity were 

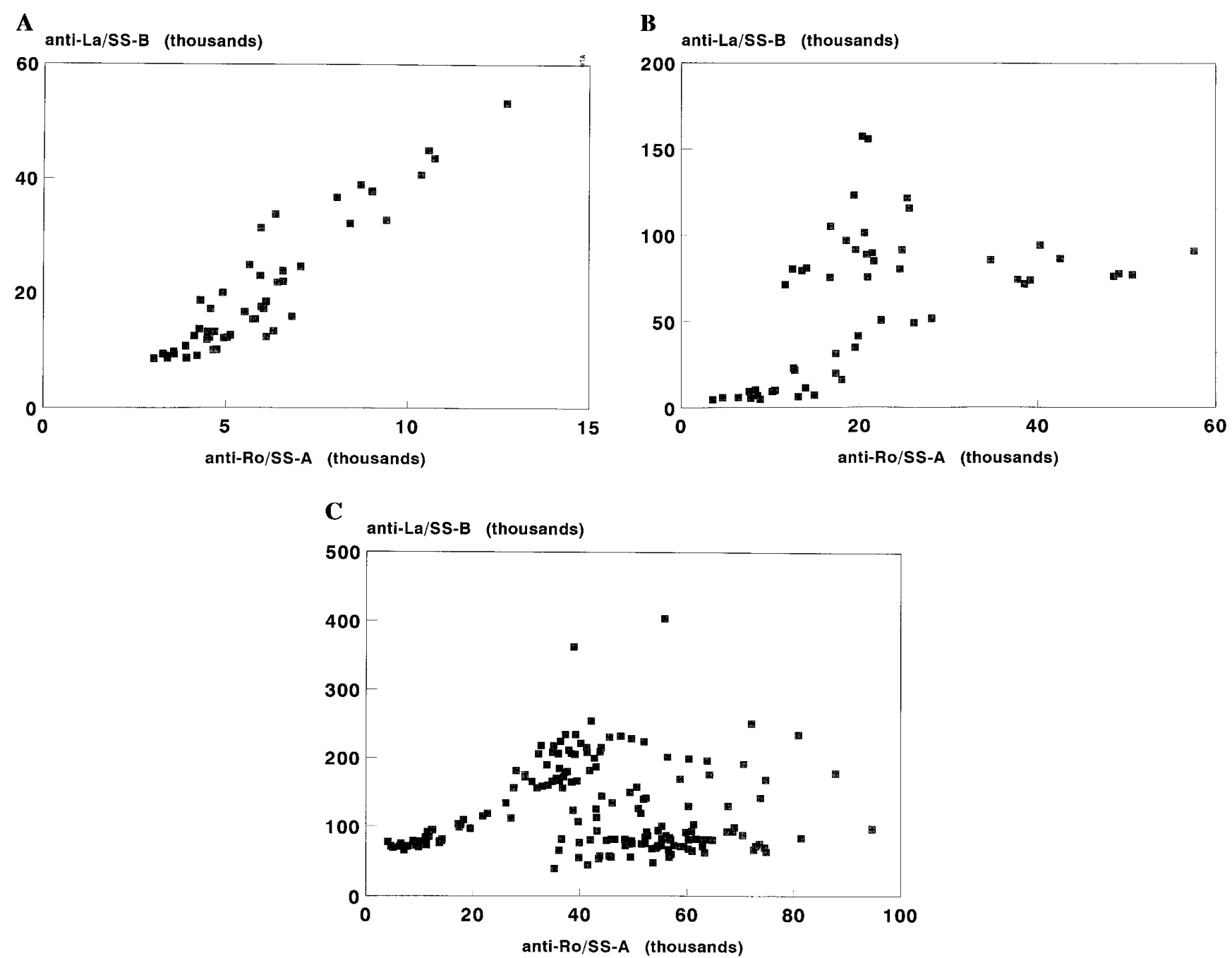

Figure 2. Relationship between anti-Ro/SS-A and anti-La/SS-B antibody levels. Calculation of the correlation between anti-Ro/SS-A and anti-La/SS-B antibody levels in all samples from the three patients depicted in Figure 1 yielded contrasting results. Depicted are anti-Ro/SS-A reactivities on the $\mathrm{x}$-axis versus anti-La/SS-B reactivities on the y-axis. Each box represents one sample taken during the observation period. Correlation was calculated using Spearman's Rank Correlation test. (A) In patient $\mathrm{B}$ there is a clear correlation between both responses throughout the observation period $(r=0.88, P<0.0001)$. (B) In patient $G$ correlation although significant is evidently less $(r=0.63, P<0.0001)$ while in patient $D(C)$ there is no correlation at all $(r=-0.04, P=0.58)$.

obtained. None of the patients became positive for one of the two antibodies studied during the observation period. Only in one patient did anti-Ro/SS-A antibodies ever disappear from the circulation after a long and gradual decline.

\section{Correlation between production of anti-Ro/SS-A and anti-La/SS-B autoantibodies in patients with SLE during longterm follow-up}

Next we determined whether there was a correlation between the magnitude of both anti-scRNP (anti-Ro/ SS-A and anti-La/SS-B) responses in individual patients. Employment of the Spearman Rank Correlation test yielded the following results for the above mentioned patients (Figure 2A, B and C). Correlation between anti-Ro/SS-A and anti-La/SS-B antibody levels was most prominent in patient B $(r=0.88$,
$P<0.0001)$ (Figure 2A) while in patient $G(r=0.63$, $P<0.0001)$ (Figure 2B) and in patient D $(r=-0.04$, $P=0.58$ ) (Figure $2 \mathrm{C}$ ) correlation was less impressive or even absent. However, division of the observation period into smaller consecutive periods (each still spanning several years) revealed that anti-scRNP antibodies were strongly correlated in these patients although the level of correlation differed during distinct periods of the patient's illness (Figure 3A-F).

Results of all nine patients are summarized in Table 1: anti-Ro/SS-A and anti-La/SS-B antibody levels were significantly and strongly correlated throughout the observation period in three patients (B, E and $\mathrm{H})$. In five other patients, subdivision periods could be selected (spanning 27 to 108 months) where a strong correlation was present. Only in patient J did correlation (even for shorter periods) never exceed $r$ values of $0.57(P<0.0001)$ which, although statistically significant, could be regarded as relatively weak. In total, a 

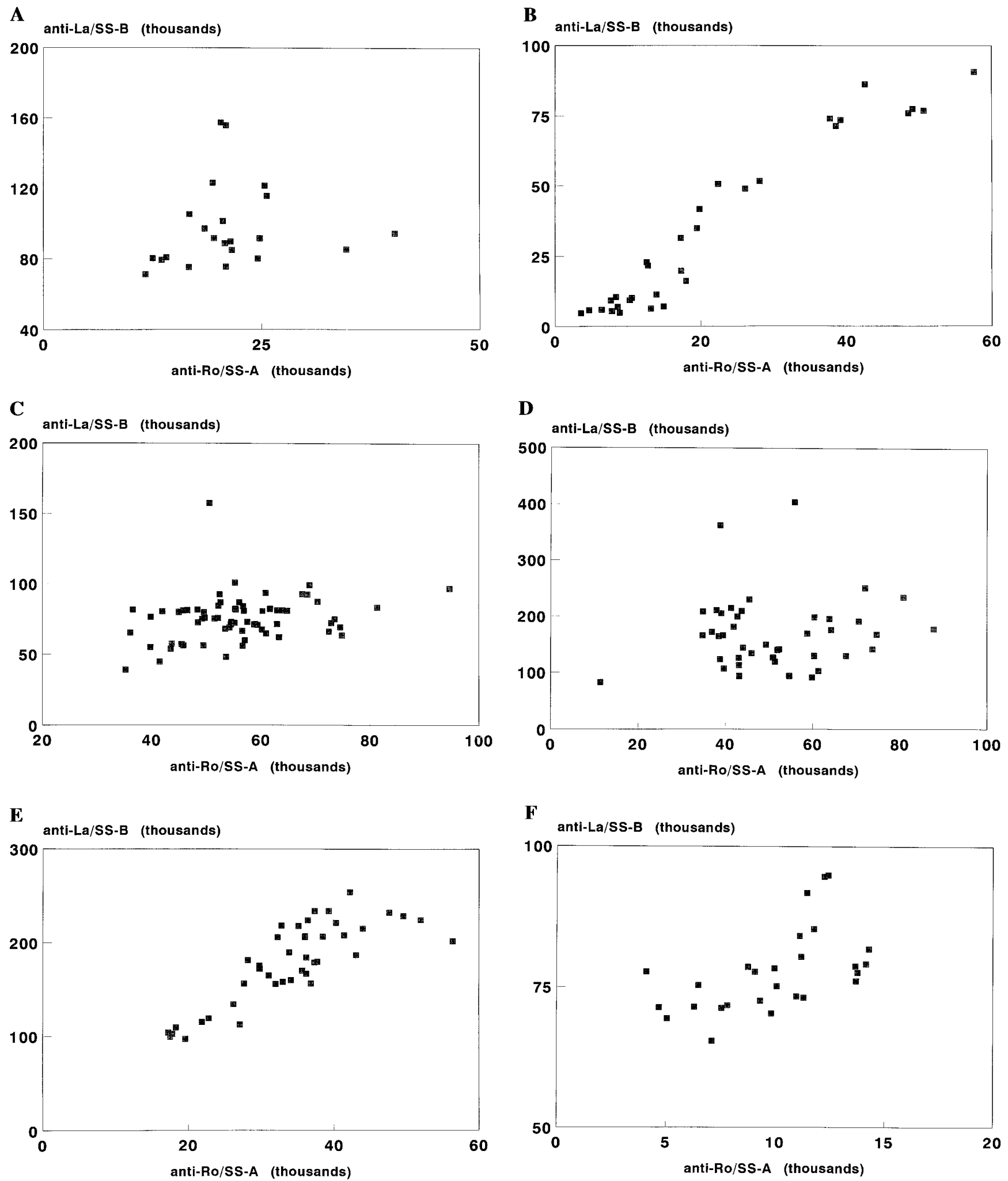

Figure 3. Relationship between anti-Ro/SS-A and anti-La/SS-B antibody levels during different consecutive periods of follow-up of patients G and D. When the observation period was dissected into smaller consecutive periods spanning several years each, periods with and without significant correlation between anti-Ro/SS-A and anti-La/SS-B antibody levels emerged in all patients studied. For example in patient $G$ there was no significant correlation during the first three years $(r=0.34$, $P=0.13$ ) (A) but a very significant one during the last six years $(r=0.94, P<0.0001)(B)$. These periods are indicated by arrows in Figure $1 B$ and $1 C$. In patient $D$ four periods were identified, spanning 46, 31, 47 and 27 months respectively. In period I (C) correlation was weak but significant $(r=0.28, P=0.02)$ while in period II (D) there was no correlation at all $(r=0.03, P=0.85)$. However in period III (E) and to a lesser degree in period IV (F) there was a significant correlation $(r=0.81, P<0.0001$ and $r=0.65, P<0.0002$ respectively). Again, all correlations were calculated using Spearman's Rank Correlation test. 
Table 1. Correlation between anti-Ro SS-A and anti-La SS-B autoantibody responses in patients with SLE during consecutive periods of follow-up

\begin{tabular}{|c|c|c|c|c|c|c|}
\hline \multirow{2}{*}{ Patients } & \multicolumn{3}{|c|}{ Periods } & \multicolumn{2}{|c|}{ Correlation } & \multirow{2}{*}{ Comment } \\
\hline & No. & Months & Samples & $r$ & $P$ & \\
\hline \multirow[t]{4}{*}{ A } & I & 27 & 51 & 0.70 & $<0.0001$ & \\
\hline & II & 20 & 21 & 0.84 & $<0.0001$ & \\
\hline & III & 54 & 37 & 0.91 & $<0.0001$ & \\
\hline & IV & 34 & 38 & 0.81 & $<0.0001$ & \\
\hline B & I & 82 & 50 & 0.88 & $<0.0001$ & \\
\hline \multirow[t]{4}{*}{ C } & I & 4 & 2 & 1.0 & 1.0 & NS \\
\hline & II & 27 & 16 & 0.78 & 0.0004 & \\
\hline & III & 20 & 16 & 0.54 & 0.03 & \\
\hline & IV & 38 & 29 & 0.05 & 0.80 & NS \\
\hline \multirow[t]{4}{*}{$\mathrm{D}$} & I & 46 & 67 & 0.28 & 0.02 & \\
\hline & II & 31 & 43 & 0.03 & 0.85 & NS \\
\hline & III & 47 & 42 & 0.81 & $<0.0001$ & \\
\hline & IV & 27 & 27 & 0.65 & 0.0002 & \\
\hline E & $\mathrm{I}$ & 63 & 25 & 0.74 & $<0.0001$ & \\
\hline \multirow[t]{2}{*}{$\mathrm{F}$} & I & 42 & 42 & 0.37 & 0.015 & \\
\hline & II & 108 & 124 & 0.88 & $<0.0001$ & \\
\hline \multirow[t]{2}{*}{$\mathrm{G}$} & I & 35 & 22 & 0.34 & 0.13 & NS \\
\hline & II & 80 & 31 & 0.94 & $<0.0001$ & \\
\hline $\mathrm{H}$ & I & 127 & 71 & 0.84 & $<0.0001$ & \\
\hline $\mathrm{J}$ & I & 187 & 98 & 0.57 & $<0.0001$ & \\
\hline Total & 21 & 1099 & 852 & & & \\
\hline
\end{tabular}

Correlation was sought between anti-scRNP antibodies by comparing longitudinal graphs of anti-Ro/SS-A with anti-La/SS-B reactivity. Visually suspected correlation was confirmed or rejected using a nonparametric Spearman Rank Correlation test. Even if there was no significant correlation between anti-scRNP antibodies during the whole follow-up period, shorter periods could be identified in which there was such a correlation. In patients $\mathrm{B}, \mathrm{E}, \mathrm{H}$ and $\mathrm{J}$ anti-scRNP antibodies were significantly correlated throughout the observation period. Patients A, C, F and G did show a (relatively low) significant correlation when all samples were tested together which could be dissected into periods where correlation was much higher or totally absent. In patient $\mathrm{D}$ a significant correlation between anti-scRNP antibodies became obviously only after dividing the follow-up into four consecutive periods. Taking all patients together there was a significant correlation between anti-Ro/SS-A and anti-La/SS-B antibodies during a total of 991 months of observation while during 108 months no such correlation could be observed.

$r=$ Correlation coefficient; NS=not significant.

significant correlation between anti-scRNP antibodies was present in 17 out of 21 periods or in 991 months of the 1,099 month cumulative observation period including all nine patients. This correlation suggests coordinated production of these antibodies.

\section{Anti-dsDNA antibody and serum immunoglobulin levels in sera of patients with SLE during longterm follow-up}

Because of the observed strong correlation between anti-Ro/SS-A and anti-La/SS-B antibody levels, it was necessary to exclude the possibility that all observed fluctuations in autoantibody responses were a result of polyclonal B cell activation. Therefore we tested all patients for anti-dsDNA antibodies in the Farr assay. Two patients (A and D) were found to produce antidsDNA antibodies during some time of the observation period. The results are presented in Figure 4 . Anti-dsDNA antibody levels fluctuated in both patients and sometimes did not exceed the cutoff value of $20 \mathrm{IU} / \mathrm{ml}$. Peak reactivities did not seem to coincide with similar peaks in anti-Ro/SS-A or antiLa/SS-B reactivity (Figure $4 \mathrm{~A}$ and $\mathrm{B}$ ). This apparent lack of correlation between anti-dsDNA and antiscRNP responses could be confirmed by performing Spearman Rank correlation tests for the already selected periods (Table 2). Although sometimes a significant correlation coefficient was found, the actual $r$ values never exceeded -0.51 and were always significantly smaller than their value for correlation between anti-Ro/SS-A and anti-La/SS-B during the same period. The only exception was period II of patient $\mathrm{D}$ where there was no correlation between the latter two responses. Furthermore, $r$ values were mostly negative but also sometimes positive (patient A, period I), an indication that a consistent correlation between anti-dsDNA and anti-scRNP responses was absent. On the other hand fluctuations in anti-scRNP antibody levels could merely be a reflection of total immunoglobulin levels. Because both autoantibody 

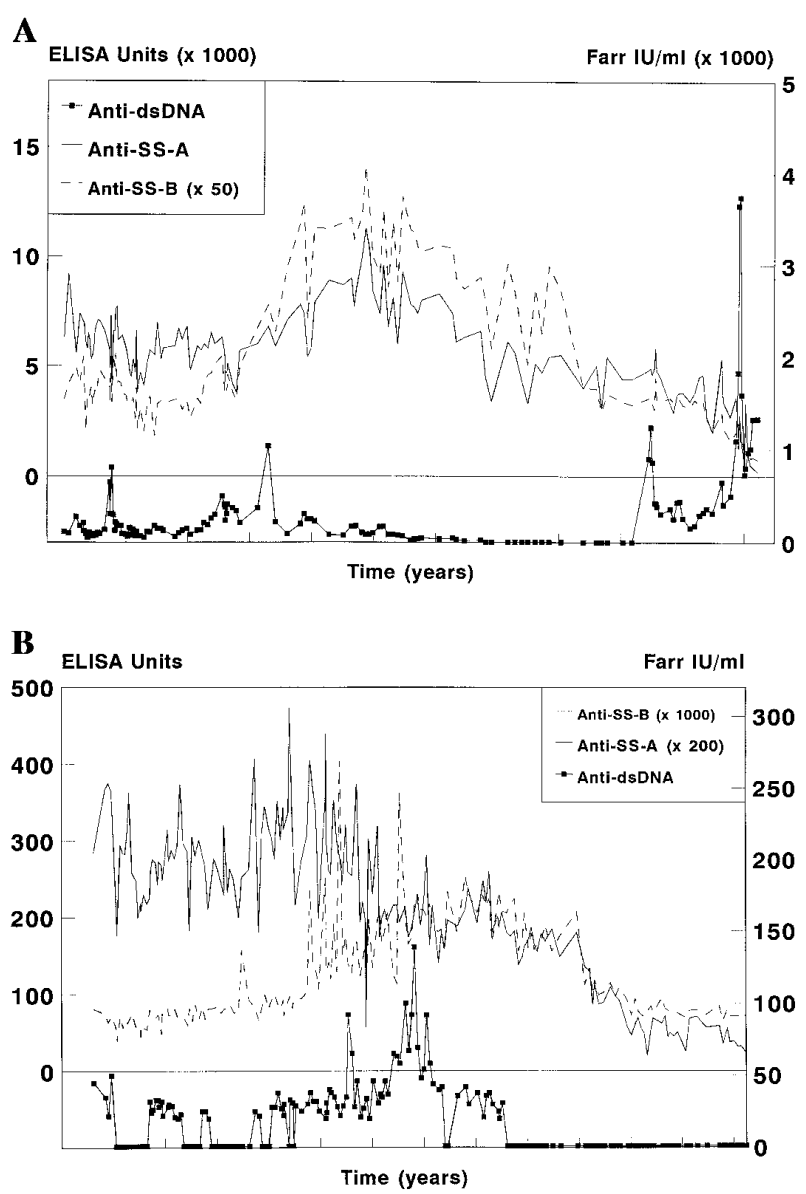

Figure 4. Development of anti-dsDNA antibody levels in time in patients $\mathrm{A}$ and D. Anti-dsDNA antibody levels during follow-up of patients A (A) and D (B). Anti-dsDNA antibody levels are expressed in Farr units on the right y-axis. For comparison anti-Ro/SS-A and anti-La/SS-B antibody levels are also presented. Their reactivity is expressed in arbitrary ELISA units on the left y-axis. The observation period (in years) is on the x-axis. Although anti-dsDNA fluctuate they do not follow the pattern of either anti-Ro/ SS-A or anti-La/SS-B antibody levels in time. In contrast to anti-scRNP antibodies, anti-dsDNA antibodies sometimes decrease to very low levels and even are below the detection limit of $20 \mathrm{IU} / \mathrm{ml}$ during several periods.

responses were previously found to be predominantly of the IgG1 subclass, we determined IgG1 levels in a limited number of sera of six patients (data not shown). There was no significant correlation between total IgG1 levels and anti-Ro/SS-A or anti-La/SS-B antibody levels.

\section{Discussion}

A correlation between the occurrence of anti-Ro/SS-A and anti-La/SS-B antibodies has long been suspected, based on non-quantitative, cross-sectional studies [33]. Our study is the first to actually demonstrate a correlation between the magnitude of both responses in individual patients. The uniqueness of this close correlation is further demonstrated by the consistent lack of correlation between anti-Ro/SS-A or anti-La/ SS-B and anti-dsDNA responses.

Recent murine studies have convincingly demonstrated that anti-Ro/SS-A and anti-La/SS-B antibodies can be the product of immunization with one particle, a ribonucleoprotein particle containing both $60 \mathrm{kDa}$ Ro/SS-A and $50 \mathrm{kDa}$ La/SS-B proteins coupled to yRNA molecules [20]. Another (52 kDa) Ro/SS-A protein exists which is probably not a constituent of the scRNP particles [34]. There is some resemblance between the above studies and those by Mamula and coworkers, who elegantly demonstrated the prerequisites for the induction of true autoantibodies against the different small nuclear ribonucleoproteins (snRNPs) in immunized mice $[18,35]$. They performed immunization experiments in mice with autologous and heterologous small nuclear ribonucleoproteins and their constituent proteins [18, 35, 36]. Immunizations with foreign snRNPs alone did indeed lead to the production of autoantibodies to snRNPs, yet not to a $T$ cell response to autologous snRNPs. To induce the latter, co-immunization with self proteins was found to be necessary. Based on these results, Mamula has recently proposed a model of autoantibody production wherein a crucial role in eliciting autoimmunity is reserved for priming of autoreactive $B$ cells with foreign crossreactive antigen, together with autoantigen to eventually activate autoreactive $\mathrm{CD}^{+}{ }^{+} \mathrm{T}$ cells [19]. The autoantigen drives the autoantibody response in this model.

Together with our studies in humans there is now strong evidence to support a central role for small cytoplasmic ribonucleoprotein particles (scRNPs) in eliciting and sustaining anti-Ro/SS-A and anti-La/ SS-B responses. Such a model of scRNP driven antiRo/SS-A and anti-La/SS-B responses elegantly explains why both antibodies are so often found together in one patient. It also explains why the magnitude of anti-Ro/SS-A and anti-La/SS-B antibody responses is correlated during most of the observation periods in individual patients: when one antigen stimulates both B cell populations their responses should mimic each other. To our knowledge, our study is the first to provide evidence for the actual existence of such a mechanism of autoreactivity driven by RNA-protein particles (in this case scRNPs) in humans.

Although we found a coordinated response to Ro/SS-A and La/SS-B in 90\% of the cumulative observation period, the question remains what happens during the (rare) periods in some patients where anti-Ro/SS-A and anti-La/SS-B antibody responses do not correlate. Apparently the antigen in those periods is not a particle containing both proteins but for instance the minority of yRNA particles containing the $60 \mathrm{kDa}$ Ro/SS-A protein only [37]. The existence of such particles could also explain why in some patients anti-Ro/SS-A antibodies can occur alone. In our laboratory we never observed the opposite-anti-La/SS-B antibodies without anti-Ro/SS-A antibodies. In one instance however, the lack of correlation in a certain period was clearly due to the development of a peak reactivity of anti-La/SS-B antibodies without change 
Table 2. Correlation between anti-scRNP and anti-dsDNA autoantibody responses in patients with SLE during consecutive periods of follow-up

\begin{tabular}{|c|c|c|c|c|c|}
\hline \multirow{2}{*}{ Patients } & \multirow{2}{*}{ Period } & \multicolumn{3}{|c|}{ Correlation } & \multirow{2}{*}{ Comment } \\
\hline & & Test & $r$ & $P$ & \\
\hline \multirow[t]{8}{*}{ A } & \multirow[t]{2}{*}{ I } & Ro vs DNA & 0.25 & 0.08 & \multirow{2}{*}{ NS } \\
\hline & & La vs DNA & 0.38 & 0.006 & \\
\hline & \multirow[t]{2}{*}{ II } & Ro vs DNA & -0.21 & 0.37 & NS \\
\hline & & La vs DNA & -0.08 & 0.75 & NS \\
\hline & \multirow[t]{2}{*}{ III } & Ro vs DNA & -0.005 & 0.98 & NS \\
\hline & & La vs DNA & 0.035 & 0.87 & NS \\
\hline & \multirow[t]{2}{*}{ IV } & Ro vs DNA & -0.39 & 0.025 & \\
\hline & & La vs DNA & -0.51 & 0.003 & \\
\hline \multirow{8}{*}{$\mathrm{D}$} & \multirow[t]{2}{*}{ I } & Ro vs DNA & -0.23 & 0.17 & NS \\
\hline & & La vs DNA & 0.12 & 0.49 & NS \\
\hline & \multirow[t]{2}{*}{ II } & Ro vs DNA & -0.35 & 0.027 & \\
\hline & & La vs DNA & 0.25 & 0.12 & NS \\
\hline & \multirow[t]{2}{*}{ III } & Ro vs DNA & -0.09 & 0.76 & NS \\
\hline & & La vs DNA & -0.20 & 0.47 & NS \\
\hline & \multirow[t]{2}{*}{ IV } & Ro vs DNA & NT & NT & NT \\
\hline & & La vs DNA & NT & NT & NT \\
\hline
\end{tabular}

Correlation was sought between anti-scRNP antibody responses and anti-dsDNA responses using the method described for Table 1. A significant correlation between anti-Ro/SS-A and anti-dsDNA antibody levels was present in only 2 out of 8 periods in these two patients. Correlation coefficients varied between -0.39 and -0.35 which is very weak compared to correlation between anti-Ro/SS-A and anti-La/SS-B. The same applies to calculated correlations between anti-La/SS-B and anti-dsDNA antibody levels, coefficients varying between -0.51 and 0.38 . During the last period of serum sampling in patient D anti-dsDNA antibody levels were persistently below the cutoff level of $20 \mathrm{IU} / \mathrm{ml}$ which precluded statistical analysis.

$\mathrm{R}=$ anti-Ro/SS-A; La=anti-La/SS-B; DNA=anti-dsDNA; $r=$ correlation coefficient; NS=Not significant; NT=not tested due to lack of data (anti-dsDNA levels below $20 \mathrm{IU} / \mathrm{ml}$ ).

in the anti-Ro/SS-A antibody titre. Which antigen stimulates such an La/SS-B restricted reactivity remains to be elucidated, although several ribonucleoprotein particles (all RNA-polymerase-III transcripts) containing only La/SS-B protein exist. Still, these particles seem not to be able to abrogate tolerance to the La/SS-B protein in the absence of anti-Ro/SS-A antibodies, thus never giving rise to anti-La/SS-B antibodies alone in a patient. Unfortunately, data on drug therapy for these patients were incomplete precluding an analysis of the role of immunosuppressive therapy in the disturbance of the coordinated anti-Ro/ SS-A and anti-La/SS-B responses. However, it could be concluded from the clinical data that our patients very infrequently experienced a major exacerbation of their disease which makes the extended use of aggressive therapy very unlikely.

Although molecular mimicry thus may play a role in the induction of autoimmune responses, our studies demonstrate that the maintenance of anti-Ro/ SS-A and anti-La/SS-B autoantibodies is possibly autoantigen driven. Murine studies implicate the scRNPs containing both Ro/SS-A and La/SS-B proteins as common autoantigen in both responses.

\section{Acknowledgements}

The authors wish to thank Vera Huysen for maintaining the collection of stored sera and Dr Hylkema for stimulating discussions.

\section{References}

1. Tan E.M., Schur P.H., Carr R.I., Kunkel H.G. 1966. Deoxyribonucleic acid (DNA) and antibodies to DNA in the serum of patients with Systemic Lupus Erythematosus. J. Clin. Invest. 45: 1732-1740

2. Tan E.M., Cohen A.S., Fries J.S., Masi A.T., McShane D.J., Rothfield N.F., Green Shaller J., Talal N., Winchester R.J. 1982. The 1982 revised criteria for the classification of systemic lupus erythematosus. Arthritis Rheum. 25: 1271-1277

3. Tan E.M. 1989. Antinuclear antibodies: Diagnostic markers for autoimmune diseases and probes for cell biology. Adv. Immunol. 44: 93-152

4. Koffler D., Schur P.M., Kunkel H.G. 1967. Immunological studies concerning the nephritis of SLE. J. Exp. Med. 126: 607-613

5. Brinkman K., Termaat R.-M., Berden J.H.M., Smeenk R.J.T. 1990. Anti-DNA antibodies and lupus nephritis: the complexity of crossreactivity. Immunol. Today 11: 232-234

6. Alspaugh M.A., Maddison P.J. 1979. Resolution of the identity of certain antigen-antibody systems in systemic lupus erythematosus and Sjögren's Syndrome: an interlaboratory collaboration. Arthritis Rheum. 22: 796-798

7. Meilof J.F. 1992. Autoantibodies against small cytoplasmic ribonucleoproteins: the anti-Ro/SS-A and anti-La/SS-B autoimmune response. A review of autoantibody detection, autoantigen composition, autoantibody-disease associations and possible etiologic mechanisms. Rheumatol. Int. 12: 129-140 
8. Scott J.S., Maddison P.J., Taylor P.V., Esscher E., Scott O., Skinner R.P. 1983. Connective-tissue disease, antibodies to ribonucleoprotein, and congenital heart block. N. Engl. J. Med. 309: 209-212

9. Meilof J.F., Frohn-Mulder I.M.E., Stewart P.A., Szatmari A., Hess J., Veldhoven C.H.A., Smeenk R.J.T., Swaak A.J.G. 1993. Maternal autoantibodies and congenital heartblock. No evidence for a unique congenital heart block-associated anti-Ro/SS-A autoantibody profile. Lupus 2: 239-246

10. Dziarski R. 1988. Autoimmunity: Polyclonal activation or antigen induction. Immunol. Today 9: 340-342

11. Zouali M., Jefferis M., Eyquem A. 1984. IgG subclass distribution of autoantibodies to DNA and to nuclear ribonucleoproteins in autoimmune diseases. Immunology 51: 595

12. Rubin R.L., Tang F.-L., Chan E.K.L., Pollard K.M., Tsay G., Tan E.M. 1986. IgG subclasses of autoantibodies in systemic lupus erythematosus, Sjögren's syndrome, and drug-induced autoimmunity. J. Immunol. 137: 2528-2534

13. Meilof J.F., Hebeda K.M., de Jong J., Smeenk R.J.T. 1992. Analysis of heavy and light chain use of Lupus associated anti-La/SS-B and anti-Sm autoantibodies reveals two distinct underlying immunoregulatory mechanisms. Res. Immunol. 143: 711-720

14. Chan E.K.L., Tan E.M. 1987. Human autoantibodyreactive epitopes of SS-B/La are highly conserved in comparison with epitopes recognized by murine monoclonal antibodies. J. Exp. Med. 166: 1627-1640

15. St Clair E.W., Burch J.A. Jr., Ward M.M., Keene J.D., Pisetsky D.S. 1990. Temporal correlation of antibody responses to different epitopes of the human $\mathrm{La}$ autoantigen. J. Clin. Invest. 85: 515-521

16. Scofield R.H., Harley J.B. 1991. Autoantigenicity of Ro/SSA antigen is related to a nucleocapsid protein of vesicular stomatitis virus. Proc. Natl. Acad. Sci. USA 88: 3343-3347

17. Weng Y.M., McNeilage J., Topfer F., McCluskey J., Gordon T. 1993. Identification of a human-specific epitope in a conserved region of the La/SS-B autoantigen. J. Clin. Invest. 92: 1104-1108

18. Fatenejad S., Mamula M., Craft J. 1993. Role of intermolecular/intrastructural B- and T-cell determinants in the diversification of autoantibodies to ribonucleoprotein particles. Proc. Natl. Acad. Sci. USA 90: $12010-12016$

19. Mamula M.J., Janeway C.A. Jr. 1993. Do B cells drive the diversification of immune responses? Immunol. Today 14: 151-153

20. Topfer F., Gordon T., McCluskey J. 1995. Intra- and intermolecular spreading of autoimmunity involving the nuclear self-antigens La (SS-B) and Ro (SS-A). Proc. Natl. Acad. Sci. USA 92: 875-879

21. Hardin J.A. 1986. The lupus autoantigens and the pathogenesis of systemic lupus erythematosus. Arthritis Rheum. 29: 457-460

22. Harley J.B., Alexander E.L., Bias W.B., Fox O.F., Provost T.T., Reichlin M., Yamagata H., Arnett F.C. 1986. Anti-Ro(SS-A) and anti-La(SS-B) in patients with Sjögren's syndrome. Arthritis Rheum. 29: 196-206
23. Hendrick J.P., Wolin S.L., Rinke J., Lerner M.R., Steitz J.A. 1981. Ro small cytoplasmic ribonucleoproteins are a subclass of La ribonucleoproteins: further characterization of the Ro and La small ribonucleoproteins from uninfected and infected mammalian cells. Mol. Cell. Biol. 1: 1138-1149

24. Wolin S.L., Steitz J.A. 1984. The Ro small cytoplasmic ribonucleoproteins: identification of the antigenic protein and its binding site on the Ro RNAs. Proc. Natl. Acad. Sci. USA 81: 1996-2000

25. Boire G., Craft J. 1990. Human Ro ribonucleoprotein particles: characterization of the native structure and stable association with the La polypeptide. J. Clin. Invest. 85: 1182-1190

26. Swaak A.J.G., Groenwold J., Aarden L.A., Statius van Eps L.W., Feltkamp T.E.W. 1982. Prognostic value of anti-dsDNA in SLE. Ann. Rheum. Dis. 41: 388-395

27. Veldhoven C.H.A., Meilof J.F., Huisman J.G., Smeenk R.J.T. 1992. The development of a quantitative assay for the detection of anti-Ro/SS-A and anti-La/SS-B autoantibodies using purified recombinant proteins. J. Immunol. Methods 151: 177-189

28. Deutscher S.L., Harley J.B., Keene J.D. 1988. Molecular analysis of the 60-kDa human Ro ribonucleoprotein. Proc. Natl. Acad. Sci. USA 85: 9479-9483

29. Chambers J.C., Kenan D., Martin B.J., Keene J.D. 1988. Genomic structure and amino acid sequence domains of the human La autoantigen. J. Biol. Chem. 263: 18043-18051

30. Aarden L.A., Smeenk R. Measurements of antibodies specific for DNA. In Immunological Methods, volume II, edited by Lefkovits I., Pernis B. Academic Press, New York, 1981, pp. 75-82

31. Feltkamp T.E.W., Kirkwood T.B.L., Maini R.N., Aarden L.A. 1988. The first international standard for antibodies to dsDNA. Ann. Rheum. Dis. 47: 740-746

32. Meilof J.F., Van der Lelij A.G., Rokeach L.A., Hoch S.O., Smeenk R.T.J. 1993. Autoimmunity and filariasis. Autoantibodies against cytoplasmic cellular proteins in sera of patients with Onchocerciasis. J. Immunol. 151: 5800-5809

33. Craft J.E., Hardin J.A. 1987. Linked sets of antinuclear antibodies: what do they mean? J. Rheumatol. 14 S13: 106-109

34. Kelekar A., Saitta M.R., Keene J.D. 1994. Molecular composition of Ro small ribonucleoprotein complexes in human cells. Intracellular localization of the 60- and 52-kD proteins. J. Clin. Invest. 93: 1637-1644

35. Mamula M.J., Fatenejad S., Craft J. 1994. B cells process and present lupus autoantigens that initiate autoimmune $\mathrm{T}$ cell responses. J. Immunol. 152: 1453-1461

36. Bockenstedt L.K., Gee R.J., Mamula M.J. 1995. Self-peptides in the initiation of lupus autoimmunity. J. Immunol. 154: 3516-3524

37. Mamula M.J., Silverman E.D., Laxer R.M., Bentur L., Isacovics B., Hardin J.A. 1989. Human monoclonal anti-La antibodies. The La-protein resides on a subset of Ro particles. J. Immunol. 143: 2923-2928 